\title{
EFFECT OF VACUUM POLARIZATION ON THE EXCITATION OF HYDROGEN ATOM BY ELECTRON IMPACT
}

\author{
SUJATA BHATTACHARYYA \\ Gokhale College, Calcutta-20 \\ INDIA \\ LALI CHATTERJEE AND T. ROY \\ Physics Department \\ Jadavpur University, Calcutta-32 \\ (Received February 11, 1980 and in revișed form April 30, 1980)
}

ABSTRACT. The vacuum polarization potential is included in the interaction Hamiltonian for $1 \mathrm{~S}-2 \mathrm{~S}$ excitation of the hydrogen atom by electron impact. The excitation amplitude calculated field theoretically is found to be lowered by

$$
0.47 t^{2} /\left(t^{2}+9\right)^{3}
$$

where $t^{2}=4|P-Q|^{2}, P$ and $Q$ being the momenta of the incident and scattered electrons respectively.

KEY WORDS AND PHRASES. Poxarization Potential, Atomec and Molecular Collesions. 1980 MATHEMATICS SUBJECT CLASSIFICATION CODES. $81645,81655$.

1. INTRODUCTION.

This paper is accessible to readers familiar with our reference [1]. The generalist reader interested in this area may start with the bibliography given there and in [2].

The cross-section for excitation of ground state hydrogen atoms to $2 \mathrm{~S}$ metastable state on electron impact has been the subject of extensive theoretical investigation. Very recently it has been calculated by Kingston, Fen and Burke [3] 
with close-coupling approximation showing good agreement with the measurements of Williams and Willis [4]. Callaway, McDowell and Morgan [5] did the calculation in a hybrid pseudostate close-coupling distorted wave model. Here also agreement with the experimental result is good. Roy and Bhattacharyya [2] tried the problem in a field theoretic way and compared the result with the then available experimental result of Stebbing, Fite, Hummer, and Brackmann [1]. Even now this simple scattering problem in atomic physics is not a closed chapter. There is room for theorization and approximation.

Although vacuum polarization gives a very small correction to the Coulomb potential, it is of interest to see its influence on the excitation amplitude. In the muonic atom the effect of vacuum polarization has been extensively studied and found to affect the energy levels [6]. In non-relativistic approximation vacuum polarization is known to affect the hydrogen $S-$ states and in particular the $2 \mathrm{~S}$ level is lowered by $27 \mathrm{MHz}$ relative to the $2 \mathrm{P}-$ level. As such it is not out of place to look for the effect of vacuum polarization on the excitation amplitude of the hydrogen atom.

\section{MATHEMATICAL FORMALISM.}

Quantum electrodynamics predicts deviation from Coulomb potential behavior near a point charge. The potential energy of a negative charge -e located at a distance $r$ from another point charge is not given entirely by the Coulomb expression $\lambda$ :

$$
v_{1}=e^{2} \int \frac{\rho(r) \rho\left(r^{\prime}\right)}{\left|r-r^{\prime}\right|} d^{3} r d^{3} r^{\prime}
$$

There is a correction of order $\alpha$, the Uehling potential. It arises from the polarization of the vacuum of the virtual electrons of mass $m$ and is given by [6]

$$
v_{2}=e^{2} \int \frac{\rho(r) \rho\left(r^{\prime}\right)}{\left|r-r^{\prime}\right|}\left\{\frac{\alpha}{\pi} z_{0}\left(r-r^{\prime}\right)\right\} d^{3} r d^{3} r^{\prime}
$$

where

$$
z_{0}(R)=\frac{2}{3}\{\ln (m R)+\gamma\}+\frac{5}{2}-\frac{1}{2} \pi(m R)
$$

here $\gamma=0.57721$ is the Euler's constant

and $\rho(r)$ is the charge density of the electron field

$$
\rho(r)=\int \psi^{*}(r) \dot{\psi(r)} d^{3} r
$$


The amplitude for $1 \mathrm{~S}$ - 2S excitation of the hydrogen atom by electron impact due to static Coulomb interaction $v_{1}$ as computed by Roy and Bhattacharyya [2] is

$$
\begin{aligned}
& \mathrm{M}_{\mathrm{fi}}=4 \int \sum_{\mathrm{ab}, \mathrm{cd}} \mathrm{g}_{\mathrm{cd}}^{{ }^{*} \mathrm{rr}}\left(\ell \ell^{\prime} \mathrm{k}_{3}\right) \mathrm{g}_{\mathrm{ab}} \mathrm{s}^{\prime}\left(k \mathrm{k}^{\prime} \mathrm{k}_{3}\right) \frac{\delta^{3}\left(\mathrm{k}-\ell+\mathrm{k}^{\prime}-\ell^{\prime}\right)}{\left|\mathrm{k}^{\prime}-\ell^{\prime}\right|} \times \\
& x_{r}^{*} x_{s} x_{r^{\prime}}^{*} x_{s^{\prime}} \quad d^{3} k d^{3} l d^{3} k^{\prime} d^{3} \ell^{\prime} d^{3} k_{3}
\end{aligned}
$$

which can be written as

$$
\begin{aligned}
& M_{f i}=4 \int \sum_{a b, c d} g_{c d}^{{ }^{r} r^{\prime}}\left(\ell \ell^{\prime} k_{3}\right) g_{a b}^{s^{\prime}}\left(k k^{\prime} k_{3}\right) \delta^{3}\left(k-\ell+k^{\prime}-\ell^{\prime}\right) \frac{\exp \left\{i\left(k^{\prime}-\ell^{\prime}\right) R\right\}}{|R|} \times \\
& x_{r}^{*} x_{s} x_{r^{\prime}}^{*} x_{s^{\prime}} d^{3} k d^{3} l d^{3} k^{\prime} d^{3} \ell^{\prime} d^{3} k_{3} d^{3} R \cdot \lambda
\end{aligned}
$$

We can proceed in a similar way to compute contributions of the vacuum polarization term $v_{2}$ towards the amplitude

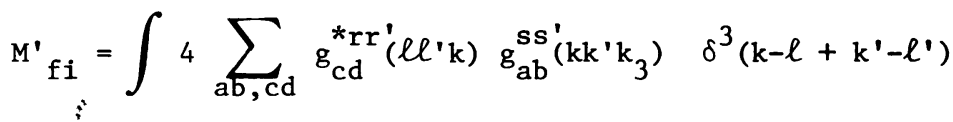

$$
\begin{aligned}
& \times \frac{\alpha}{\pi} z_{0}(R) \frac{\exp \left\{i\left(k^{\prime}-\ell^{\prime}\right) R\right\}}{|R|} x_{r}^{*} x_{r^{\prime}} x_{s} x_{s^{\prime}} \\
& \times d^{3} k d^{3} \ell d^{3} k^{\prime} d^{3} \ell^{\prime} d^{3} R d^{3} k_{3}
\end{aligned}
$$

The total amplitude $W$ for the process in the co-ordinate space is obtained by adding $\mathrm{M}_{\mathrm{fi}}$ to $\mathrm{M}^{\prime} \mathrm{fi}_{\mathrm{i}}$, and integrating over $\mathrm{k}, \mathrm{k}^{\prime}, \ell, \ell^{\prime}$, and $\mathrm{k}_{3}$ we get as in the previous paper [2]

$$
W=4 \int \sum_{a b, c d} \Phi_{c d}(x, x+R) \Phi_{a b}(x, x+R) \frac{1}{|R|}\left(1+\frac{\alpha}{\pi} z_{0}(R)\right) \delta^{3}\left(P_{c}-O_{c}\right) d^{3} x d^{3} R
$$

$\Phi_{a b}(X, X+R)$ and $\Phi_{c d}(X, X+R)$ are initial and final state solutions respectively of the Schrodinger equation for the combined system of hydrogen atom and a free electron.

Writing these in a slater determinant form, equation (2.8) becomes

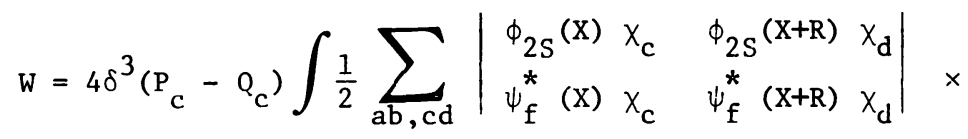

$$
\begin{aligned}
& \times\left|\begin{array}{cccc}
\phi_{1 S}(X) & x_{a} & \phi_{1 S}(X+R) & x_{b} \\
\psi_{i}(X) & x_{a} & \psi_{i}(X+R) & x_{b}
\end{array}\right| \frac{1}{|R|}\left(1+\frac{\alpha}{\pi} z_{0}(R)\right) d^{3} x d^{3} R
\end{aligned}
$$

where 
$\phi_{1 S}(X), \phi_{2 S}(X)$ are respectively the ground state and $2 S$ excited state wave functions of the hydrogen atom. $\psi_{i}(x)$ and $\psi_{f}(x)$ are the plane wave solutions for the incident and scattered electrons respectively. After integrating over $X$ and $R$ (all quantities are in atomic units) and taking for Pauli spin vector

$$
\sum_{a b} x_{a} x_{b}=\sum_{a b} \delta_{a b}=2,
$$

we obtain

$$
\mathrm{W}=4\left[\left(\mathrm{~A}_{0}+\mathrm{B}_{0}+\mathrm{C}_{0}+\mathrm{D}_{0}\right)+\frac{\alpha}{\pi}\left(\mathrm{A}_{1}+\mathrm{B}_{1}+\mathrm{C}_{1}+\mathrm{D}_{1}\right)\right]
$$

With $P$ and $Q$ as the momenta of the incident and scattered electrons, and

$$
t^{2}=4|P-Q|^{2}, \quad I_{0}=0.375 I_{D}\left(t^{2}+9\right) / t^{2}, \quad I_{D}=512 t^{2} / \sqrt{2}\left(t^{2}+9\right)^{3},
$$

we obtain

$$
\begin{aligned}
& A_{0}=\left(4 \pi I_{D}\right) \frac{4}{t^{2}}, \quad B_{0}=\frac{4 \pi I_{D}}{Q^{2}+1}, \\
& C_{0}=4 \pi I_{D}\left[\frac{4}{4 P^{2}+9}-\frac{9\left(t^{2}+9\right)}{t^{2}\left(t^{2}+9\right)^{2}}\right], \\
& D_{0}=4 I_{D}\left[1.444-1.034 \frac{\left(t^{2}+9\right)}{t^{2}}\right] ; \\
& A_{1}=I_{D} \int \exp [i(P-Q) R] \frac{Z_{0}(R)}{|R|} d^{3} R, \\
& B_{1}=I_{D} \int \exp [i Q R-|R|] \frac{Z_{0}(R)}{|R|} d^{3} R, \\
& C_{1}=\int\left(I_{D}-I_{0} R\right) \exp \left[i P R-\frac{3}{2}|R|\right] \frac{z_{0}(R)}{|R|} d^{3} R, \\
& D_{1}=\int\left(I_{D}-I_{0} R\right) \exp \left[-\frac{3}{2}|R| \mid \frac{z_{0}(R)}{|R|} d^{3} R\right.
\end{aligned}
$$

Putting $Z_{0}(R)$ from (2.3) in $A_{1}$ and knowing that $R$ varies from 0 to $\lambda$ (the Compton wave length for electrons), integral $A_{1}$ becomes

$$
A_{1}=4 \pi I_{D} \frac{\lambda^{2}}{3} \cdot \frac{1}{2}(2 \ln \lambda-1)
$$


$\lambda$ being very small (45.778 $\times 10^{-3}$ in a.u.) we have approximated for low energy encounter

$$
\cos (P-Q) \lambda=1 \quad \text { and } \quad \sin (P-Q) \lambda=(P-Q) \lambda
$$

To integrate $B_{1}, C_{1}$, and $D_{1}$ we have taken on $1 y$ the first term in the expansions of $\exp (-R)$ and $\exp \left(-\frac{3}{2}|R|\right)$ and we find

$$
A_{1}=B_{1}=C_{1}=D_{1}
$$

Hence the total amplitude for the process

$$
\mathrm{W}=4\left[\mathrm{~A}_{0}+\mathrm{B}_{0}+\mathrm{C}_{0}+\mathrm{D}_{0}+\frac{8 \lambda^{2} \alpha}{3}(2 \ln -1) \mathrm{I}_{\mathrm{D}}\right]
$$

\section{CONCLUSION.}

From equation (2.14) we find that vacuum polarization reduces the Coulomb amplitude for $1 \mathrm{~S}-2 \mathrm{~S}$ excitation of hydrogen atom by

$$
10.6 \lambda^{2} \alpha(2 \ln \lambda-1) I_{D}=-0.47 \frac{t^{2}}{\left(t^{2}+9\right)^{3}}
$$

For small momentum transfer this reduction becomes $-0.47 t^{2} / 729$ while for high momentum transfer its value is $-0.47 / \mathrm{t}^{4}$. Thus change in the amplitude for low momentum transfer is greater than that for high momentum transfer. These changes are very small and give a correction of the order of 0.4 to 0.5 per cent to the differential cross-section. So there is every possibility for these to escape detection during experiment. But with the increase in the precision of the experiment we hope that these small changes may be detected in the future.

4. ACKNOWLEDGEMENT.

We would like to thank U.G.C. for financial support of this work under the project "Atomic and Molecular Collisions" at Jadavpur University.

\section{REFERENCES}

i. STEBbiNG, R.F., FITE, W.L., HUMMER, D.G., and BRACKMANN, R.T. Collisions of electrons with hydrogen atoms. V. Excitation of Metastable $2 \mathrm{~S}$ hydrogen atoms, Phys. Rev. 119 (1960), pp. 1939-1945.

2. ROY, T. and S. BHATTACHARYYA. Field theoretic approach to $1 \mathrm{~S}-2 \mathrm{~S}$ excitation in H-e collision at low energy. Lett. Nucve Cim. 9 (1974), pp. 54-58.

3. KINGSTON, A.E., FEN, W.C., BURKE, P.G. The $1 \mathrm{~S}-2 \mathrm{~S}$ and $1 \mathrm{~S}-2 \mathrm{P}$ excitation of atomic hydrogen by electron impact. J. Phys. B. $\underline{9}$ (1976), pp. 605-618. 
4. WILIIAMS, J.F. and B.A. WILLIS. Electron scattering from atomic hydrogen I. Differential cross-section for excitation of the $n=2$ states. J. Phys. B 9 (1975), pp. 1641-1669.

5. CALlAWAY, J., McDOWELL, M.R.C. and MORGAN, L.A. Inelastic differentia1 scattering of electrons by hydrogen atoms from $0.75-4$ Ryd: $n=2$ states. J. Phys. B. 9 (1976), pp. 2043-2051.

6. BROWN, L.S., CAHN, R.N., and McLERRAN, L.D. Analytical calculation to all orders in $Z$ of vacuum polarization at short distances. Phys. Rev. Lett. 33 (1974), pp. 1591-1593. 


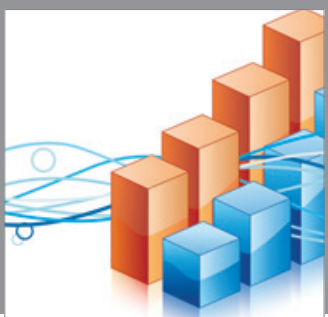

Advances in

Operations Research

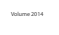

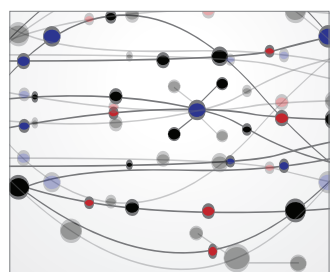

\section{The Scientific} World Journal
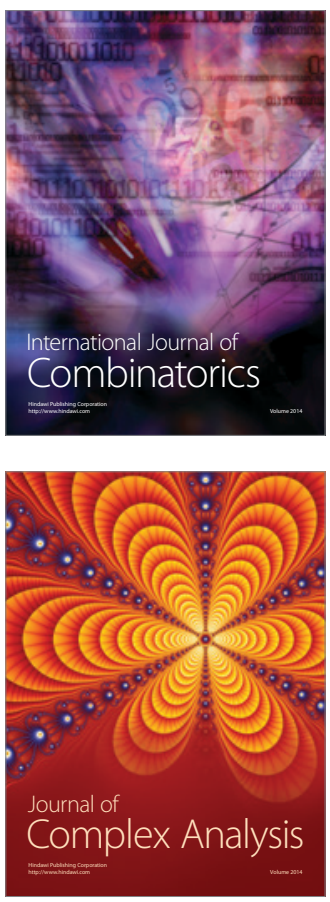

International Journal of

Mathematics and

Mathematical

Sciences
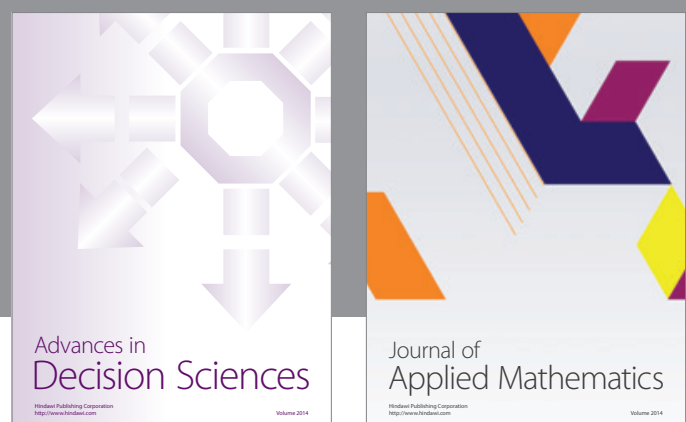

Journal of

Applied Mathematics
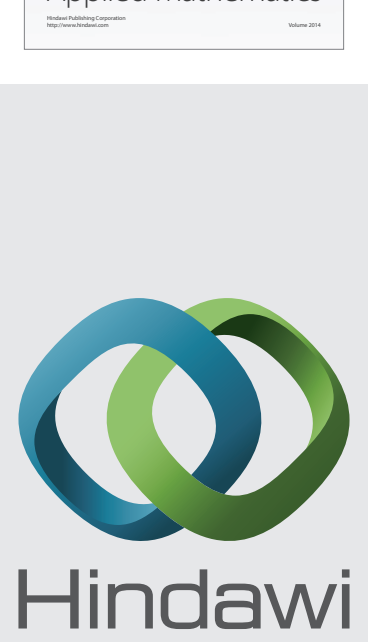

Submit your manuscripts at http://www.hindawi.com
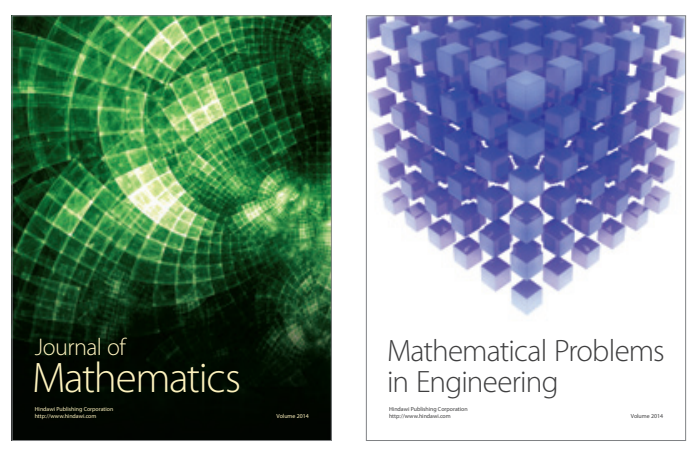

Mathematical Problems in Engineering
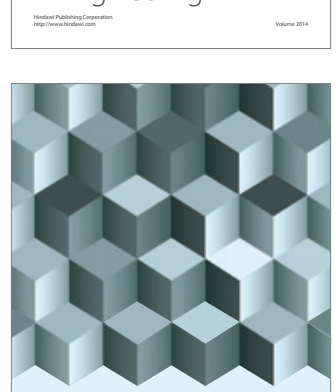

Journal of

Function Spaces
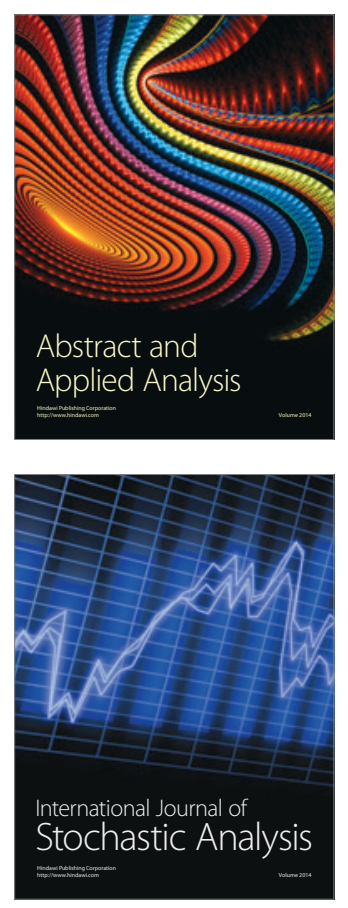

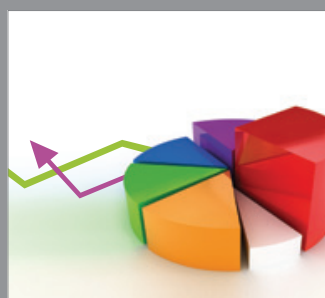

ournal of

Probability and Statistics

Promensencen
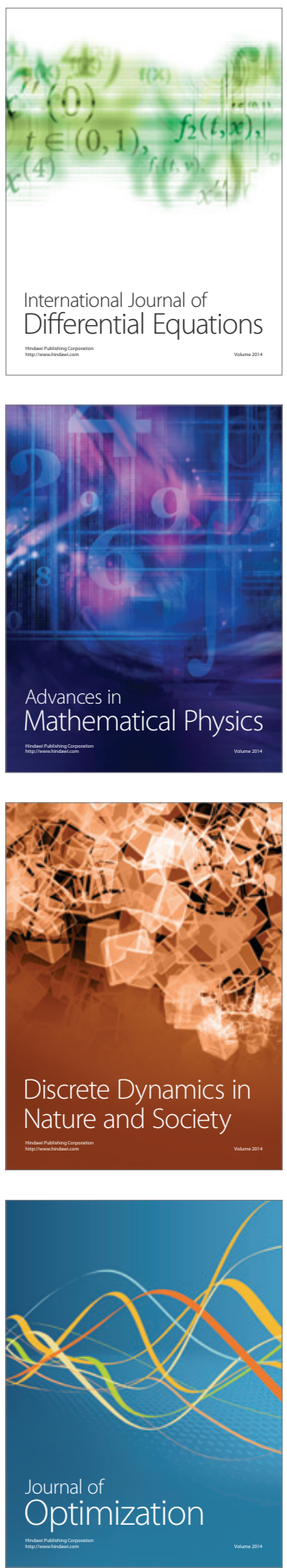